\title{
New perspectives in the treatment of hard-to-heal wounds
}

\author{
Jarosław Cwaliński, Jacek Paszkowski, Tomasz Banasiewicz
}

\section{EDITORIAL}

\begin{abstract}
Hard-to-heal wounds continue to be a challenge in the everyday surgical practice. Their treatment is timeconsuming, expensive and in many cases requires interdisciplinary assessment. Therapy option include properly selected surgical procedures and dressings combined with systemic antibiotherapy. Application of vacuum assisted closure (VAC) facilitates the evacuation of pathological discharge, reduces tissue oedema and eliminates bacterial biofilm. Complementary administration of antibiotics to control chronic infection relies today in most cases on vancomycin, ciprofloxacin or piperacillin with tazobactam, with good clinical effect.

An alternative to antibiotics against MRSA, administered at hospitals might be dalbavancin, a new generation lipoglycopeptide, which belongs to the same class as vancomycin. Introduction of dalbavancin and $\mathrm{VAC}$ might be an alternative to traditional methods of therapy.
\end{abstract}

Keywords-NPWT, Dalbavancin, biofilm, chronic wound

\section{EPIDEMIOLOGY}

$\mathbf{E}$ XTENSIVE hard-to-heal wounds represent a significant problem in everyday surgical practice. Despite many years of experience and a number of therapeutic standards effective cure is time-consuming, expensive and in many cases requires interdisciplinary involvement. Progress of surgical techniques over the last decades has contributed to an increase in total healing rate, however there is a large group of patients for whom lack of proper tissue regeneration leads to chronic organ dysfunction.

Hard-to-heal wounds are referred to as so-called "silent epidemic" which affects, according to various estimates, even $1-2 \%$ of the population in developed countries. In the US, the total number of patients hospitalized for this reason amounted to nearly 6.5 million and the total cost of treatment consumed 25 billion dollars. On the other hand, in Scandinavian countries, spending related to the treatment of chronic wounds accounts for $2-4 \%$ of the health care budget 1 [12

Multicenter experiences indicate that dealing with chronic wounds calls for a comprehensive approach and requires a holistic assessment of the problem. Adequate treatment

Manuscript received 25.07.2018; revised 22.12.2018. This work did not receive any financial support. Authors declare no conflict of interest.

Author affiliations: Department of General, Endocrinological Surgery and Gastroenterological Oncology Poznan University of Medical Sciences, Poland , (JC, JP, TB)

*Correspondence to: Jarosław Cwaliński: jaroslaw.cwalinski@gmail.com strategy depends on the improvement of local conditions as well as on the general condition of the patient. Preventing infection and keeping the injured tissue in moist setting became the basic priorities. The key issue is to separate the healing area from potential sources of pathogens, i.e. necrosis, gangrene or digestive contents in case of abdominal fistulas. Simultaneous coexistence of chronic diseases deteriorates general condition and additionally depletes the regenerative potential of damaged tissue. Cardiovascular, metabolic or renal failure as well as immunodeficiency syndromes can be a potential trigger responsible for worse healing. Other risk factors include older age, male sex, chronic steroid therapy, nicotinism and malnutrition. All but the first two can be modified in the course of treatment 10

\section{Pathogenesis of Wound formation}

However the pathophysiology of chronic wounds proves that lack of healing depends on many factors, three of them are crucial: local ischemia, infection and tissue swelling strongly inhibit wound regeneration. Most complicated cases are characterized by both synergy of these agents and their mutual enhancement. Following the microcirculation insufficiency, local inflammation and swelling occurred. Insufficient fluid supply and lack of gas diffusion facilitate bacterial colonization initiated primarily by opportunistic pathogens. As a consequence, necrosis is formed penetrating the skin, subcutaneous tissue and even deeper located structures. In addition pathological discharge accumulates on the bottom of the wound which additionally impairs its effective repair 5 The growth and survival of microorganisms within the infected area is stabilized by formation of a biofilm defined as a bacterial matrix supplemented by polymers (polysaccharides, proteins) and nucleic acids. It creates a local microenvironment that facilitates bacterial adhesion and protects colonies against adverse external factors including loss of moisture. In addition, it is a medium for signal transmission, transferring information responsible for drug resistance 6 [

The resorption of biofilm is crucial in the process of wound healing. As a barrier factor it hinders diffusion of respiratory gases and neutralizes penetration of antiseptics or anti-inflammatory solutions. By keeping the $\mathrm{pH}$ relatively stable, biofilm supports the electrochemical gradient and, due to its spatial structure, allows for the growth of both aero- 
bic and anaerobic bacteria. In hard-to-heal chronic wounds biofilm is constantly regenerated with reimplantation after 2-3 days from its primary removal. This property explains why current healing concepts recommend as a priority the permanent removal of pathological exudate from the surface of an infected wound 7 [ 8

\section{Clinical management}

The evaluation of wound healing is carried out through clinical observation focusing on detection of hematoma and inflammatory or purulent discharge. The next step is to surgically examine the wound edges and fundus in search of infected or necrotic tissues. Furthermore, if necessary, a culture is collected as well as inflammatory markers in blood serum are monitored which allows to control the effectiveness of treatment or gives an early sign that the local infection starts to develop into sepsis. 90

Wound treatment includes properly selected surgical procedures combined with systemic therapy. The key is mechanical debridement of the wound edges with removal of any pathological discharge or necrotic changes and use of appropriate antibacterial prophylaxis or even, if valuable, antibiotic therapy. Treatment of hard-to-heal injuries carries the risk of septic complications, therefore in addition to surgical procedure the use of selected systemic antibiotic should be considered. Crucial is not only the antibacterial effect of a given substance, but also the mechanism of its distribution that determines the effectiveness of penetration in the healing site. Final decision should be based on the antibiogram and must comply with clinical practice and local guidelines. 9 . 11

\section{HARD-TO-HEAL WOUNDS AND NPWT}

One of the key achievements of the last decade in the field of hard-to-heal wound therapy was the use of negative pressure as a factor able to accelerate tissue regeneration. NWPT (Negative-pressure wound therapy) or VAC (vacuumassisted closure) is done by placing a special polyurethane sponge with a hole diameter of 500 to $600 \mu \mathrm{m}$ inside the wound and covering it with foil. Finally negative pressure is generated within the dressing reaching the value from -50 to $-200 \mathrm{mmHg}$. 12

VAC therapy results in the separation of pathological discharge and reduction of residual edema. Decompression of tissues significantly improves blood perfusion and lymphatic drainage. Negative pressure effectively helps to eliminate biofilm and inhibits also its new formation. As a result of contraction of intercellular spaces and following reduction of wound surface the use of a vacuum leads to so-called micro- and macrodeformation. VAC therapy restricts local inflammation, reduces tissue hypoxia and boosts cell proliferation 13,14

Some authors also suggest its beneficial antibacterial effect, especially the limitation of Gramm-negative germs growth. Application of negative pressure favors the mechanical elimination of bacterial cells and also improves local pharmacodynamic and pharmacokinetic features of drug penetration. 14

In practical terms the effectiveness of vacuum therapy is mainly determined by two elements, i.e. the value of the generated under-pressure and its proper distribution. The value of pressure advisable in the literature ranges from 50 to $-200 \mathrm{mmHg}$ and depends on the type of wound, its location, dimensions and the degree of tissue damage. It is generally accepted that pressure from -80 to $-125 \mathrm{mmHg}$ is a compromise between sufficient removing of pathological discharge and the potential mechanical destruction of the wound surface. The problem of proper pressure distribution within the dressing is still the subject of research. There is no answer to the question about the possible difference between the value of pressure detected on the generator and its real value in different parts of dressing. The advantage of commonly used systems is the ability to individually adjust the sponge to the shape of the wound. Thereby the dressing covers the entire surface of the wound and protects it from the outside environment. Finally, the NPWT significantly minimizes the need for hospitalization, enabling further treatment in outpatient clinic 15.16

\section{NPWT VS ANTIBIOTHERAPY}

There are a number of studies evaluating the effectiveness of systemic antibiotic therapy used jointly with negative pressure therapy in the treatment of complicated wounds. The results of many analyses, although based on a relatively small group of cases, indicate that NPWT augments the therapeutic activity of intravenous injection. Rowan et al. Assessing the efficacy of vancomycin, ciprofloxacin, and piperacillin with tazobactam in the treatment of hard-to-heal wounds showed that by using a vacuum dressing, the concentration of antibiotic within the wound reaches a value of not less than $80 \%$ of plasma concentration. 11

The beneficial interaction of NPWT with systemic antibacterial treatment prompts to define a clinical algorithm allowing more effective use of both methods of therapy, especially in outpatient care. In this context, dalbavancin, a lipoglycopeptide antibiotic classified to the same group as vancomycin can increase the efficiency of wound healing. In preliminary clinical trials dalbavancin has been shown to be highly effective in the treatment of acute skin and soft tissue infections, including the elimination of methicillinresistant S. aureus (MRSA). An additional advantage is its unique pharmacodynamic profile limiting the dosage for single administration or in two doses repeated one week apart.11. 17

\section{Conclusion}

In conclusion a strategy based on surgical debridement, followed by NPWT and a single dose of dalbavancin in prophylaxis and therapy may be beneficial in a treatment of complicated wounds. However final recommendation requires further evaluation in a wider group of patients. 


\section{REFERENCES}

[1] C. K. Sen, G. M. Gordillo, S. Roy, R. Kirsner, L. Lambert, T. K. Hunt, F. Gottrup, G. C. Gurtner, and M. T. Longaker, "Human skin wounds: a major and snowballing threat to public health and the economy," Wound repair and regeneration, vol. 17, no. 6, pp. 763-771, 2009.

[2] F. Gottrup, P. Holstein, B. Jørgensen, M. Lohmann, and T. Karlsmar, "A new concept of a multidisciplinary wound healing center and a national expert function of wound healing," Archives of Surgery, vol. 136, no. 7, pp. 765-772, 2001.

[3] K. Jones, K. Fennie, and A. Lenihan, "Evidence-based management of chronic wounds." Adv Skin Wound Care., vol. 20, no. 11, pp. 591-600, 2007.

[4] M. Ferreira, P. J. Tuma, V. Carvalho, and F. Kamamoto, "Complex wounds." Clinics, vol. 61, no. 6, pp. 571-8, 2006.

[5] G. Schultz, D. Mozingo, M. Romanelli, and K. Claxton, "Wound healing and time; new concepts and scientific applications," Wound repair and regeneration, vol. 13, pp. S1-S11, 2005.

[6] A. Omar, J. Wright, G. Schultz, R. Burrell, and P. Nadworny, "Microbial biofilms and chronic wounds," Microorganisms, vol. 5, no. 1, p. 9, 2017.

[7] S. L. Percival, C. Vuotto, G. Donelli, and B. A. Lipsky, "Biofilms and wounds: an identification algorithm and potential treatment options," Advances in wound care, vol. 4, no. 7, pp. 389-397, 2015.

[8] T. Li, L. Zhang, L. Han, G. Wang, P. Yin, Z. Li, L. Zhang, Q. Guo, D. Liu, and P. Tang, "Early application of negative pressure wound therapy to acute wounds contaminated with staphylococcus aureus: An effective approach to preventing biofilm formation," Experimental and therapeutic medicine, vol. 11, no. 3, pp. 769-776, 2016.

[9] A. Sood, M. S. Granick, and N. L. Tomaselli, "Wound dressings and comparative effectiveness data," Advances in wound care, vol. 3 , no. 8 , pp. 511-529, 2014.
[10] C. E. Attinger, J. E. Janis, J. Steinberg, J. Schwartz, A. Al-Attar, and K. Couch, "Clinical approach to wounds: debridement and wound bed preparation including the use of dressings and wound-healing adjuvants," Plastic and reconstructive surgery, vol. 117, no. 7S, pp. 72S-109S, 2006

[11] M. P. Rowan, K. L. Niece, J. A. Rizzo, and K. S. Akers, "Wound penetration of cefazolin, ciprofloxacin, piperacillin, tazobactam, and vancomycin during negative pressure wound therapy," Advances in wound care, vol. 6, no. 2, pp. 55-62, 2017.

[12] K. Lambert, P. Hayes, and M. McCarthy, "Vacuum assisted closure: a review of development and current applications," European journal of vascular and endovascular surgery, vol. 29, no. 3, pp. 219-226, 2005.

[13] S. Jones, P. Banwell, and P. Shakespeare, "Advances in wound healing: topical negative pressure therapy," Postgraduate medical journal, vol. 81, no. 956, pp. 353-357, 2005.

[14] T. Li, G. Wang, P. Yin, Z. Li, L. Zhang, J. Liu, M. Li, L. Zhang, L. Han, and P. Tang, "Effect of negative pressure on growth, secretion and biofilm formation of staphylococcus aureus," Antonie van Leeuwenhoek, vol. 108, no. 4, pp. 907-917, 2015.

[15] M. Malmsjö, E. Huddleston, and R. Martin, "Biological effects of a disposable, canisterless negative pressure wound therapy system," Eplasty, vol. 14, 2014.

[16] T. Banasiewicz, M. Borejsza-Wysocki, W. Meissner, S. Malinger, J. Szmeja, T. Kościński, A. Ratajczak, and M. Drews, "Vacuumassisted closure therapy in patients with large postoperative wounds complicated by multiple fistulas," Videosurgery and other miniinvasive techniques, vol. 6, no. 3, p. 155, 2011.

[17] S. Ramdeen and H. W. Boucher, "Dalbavancin for the treatment of acute bacterial skin and skin structure infections," Expert opinion on pharmacotherapy, vol. 16, no. 13, pp. 2073-2081, 2015. 